\title{
Oral Potentially Malignant Disorders and Squamous Cell Carcinoma at the Tongue: Clinicopathological Analysis in a Thai Population
}

\author{
Anchisa Aittiwarapoj ${ }^{1}$ Rachai Juengsomjit ${ }^{1}$ \\ 1Department of Oral and Maxillofacial Pathology, Faculty of \\ Dentistry, Mahidol University, Bangkok, Thailand \\ 2Department of Oral Biology, Faculty of Dentistry, Mahidol \\ University, Bangkok, Thailand
}

Nakarin Kitkumthorn² Puangwan Lapthanasupkul ${ }^{1}$

Eur J Dent 2019;13:376-382

\begin{abstract}
Address for correspondence Puangwan Lapthanasupkul, DDS, MSc, PhD, Department of Oral and Maxillofacial Pathology, Faculty of Dentistry, Mahidol University, 6 Yothi Street, Bangkok 10400, Thailand (e-mail: puangwan.lap@mahidol.ac.th).
\end{abstract}

\begin{abstract}
Objective Tongue is regarded as one of the common sites of oral squamous cell carcinoma (OSCC). This study aimed to determine the prevalence and clinicopathological profile of OSCC and oral potentially malignant disorders (OPMDs) at the tongue.

Materials and Methods We retrospectively analyzed clinicopathological characteristics of 208 Thai patients diagnosed with SCC and OPMDs at the tongue in the period from 1996 to 2015. Chi-squared test was used to compare differences between patient's clinical and histopathological features.

Results Seventy-eight tongue SCC and 130 tongue OPMD cases were present over the study period. Slight male predominance was found for tongue SCC, while a slight female predominance was present for tongue OPMDs. Both tongue SCC and tongue OPMDs were mostly diagnosed in the old age patients ( $>40$ years old). The majority of tongue SCC and OPMDs occurred at the tip and lateral of the tongue. The most com-

Keywords

- cancer

- erythroplakia

- leukoplakia

- oral potentially malignant disorders

- squamous cell carcinoma

- tongue mon histologic grading of tongue SCC was well differentiated and no poor differentiation was found in this study. More than a half of tongue OPMDs showed epithelial dysplasia. Both tongue SCC and OPMDs demonstrated no significant correlation between clinical feature and histopathologic diagnosis.

Conclusions Tongue SCC and OPMDs were particularly found in the elderly population and frequently developed at the tip and lateral of the tongue. Most of tongue OPMDs, particularly all of tongue erythroplakia, exhibited epithelial dysplasia. For dental practitioners, awareness in the early detection and diagnosis of these tongue lesions should be raised.
\end{abstract}

\section{Introduction}

The tongue is the most common intraoral site for oral cancer and tongue cancer is an important public health problem in many countries. A recent global meta-analysis has shown an increase in incidence of oral tongue cancer worldwide ${ }^{1}$ As with other intraoral sites, squamous cell carcinoma (SCC) accounts for more than 95\% of tongue cancer. SCC of the tongue is considered as one of the most fatal site for oral SCC (OSCC) due to its aggressive behavior and high frequency of regional lymph node metastasis. ${ }^{2}$ Despite advanced surgical techniques and new therapeutic strategies, the mortality rate for OSCC remains high in most countries, with an overall 5 -year survival rate below $50 \% .{ }^{3}$ SCC of the tongue primarily affects older people; however, in the past decade it has been found increasingly in younger people. ${ }^{1}$ Tongue SCC in the young patients showed different clinicopathological features and prognosis in comparison to that in the older patients. ${ }^{4}$

Although the etiology of OSCC is not fully understood, it has been suggested that OSCCs preceded by precursor cells 
and likely lesions or conditions that subsequently progress to cancer. ${ }^{5}$ Several studies have shown that most of OSCCs arise from long-standing potentially malignant oral lesions. The term "oral potentially malignant disorder" (OPMD) describing oral lesions and conditions carrying a risk for malignant transformation was adopted by the World Health Organization (WHO) in $2005^{6}$ and mainly includes leukoplakia, erythroplakia, lichen planus, lupus erythematosus [LE], and oral submucous fibrosis. OPMD develops into cancer through various histopathological stages from hyperkeratosis and/ or hyperplasia to various degrees of oral epithelial dysplasia (OED), to carcinoma in situ, and eventually to invasive SCC. ${ }^{5}$ Early detection of tongue SCC at the premalignant stage is recommended to decrease the morbidity and mortality of this malignancy. Histopathologically, OED with various degree is assessed for predicting malignant transformation in this premalignant stage.

The aim of this study was to retrospectively analyze clinical and pathological features of SCC and OPMDs at the tongue in a Thai population over a 20 -year period and to evaluate whether any clinical features of SCC and OPMDs at tongue are associated with malignant and premalignant histopathology.

\section{Materials and Methods}

This study received ethical approval from the Institutional Review Board, Mahidol University. Oral biopsied reports with definitive diagnosis of OSCC and OPMDs (oral leukoplakia, oral erythroplakia, OLP, LE, and submucous fibrosis) at the tongue over a 20-year period (1996-2015) were retrieved from the Archive of Oral and Maxillofacial Pathology Department, Faculty of Dentistry, Mahidol University. The screening process of studies cases was shown in - Fig. 1. Clinical data, including patient demographics, clinical feature, and tongue subsite, were collected from hospital chart records. Clinical feature of tongue SCC was divided into three groups: ulcers (including plain ulcers and exophytic ulcerated masses), red and white lesions, and red and white lesions with ulcers. Clinical features of leukoplakia were subclassified as homogenous and nonhomogeneous leukoplakia, while erythroplakia was subclassified as smooth surface and rough surface erythroplakia. OLP and LE were clinically subdivided into three groups: white lesion (including white line and white plaque), red and white lesion, and red lesion. Based on International Classification of Diseases, Ninth Revision, Clinical

\section{Clinically suspected OPMDs or OSCC at tongue}

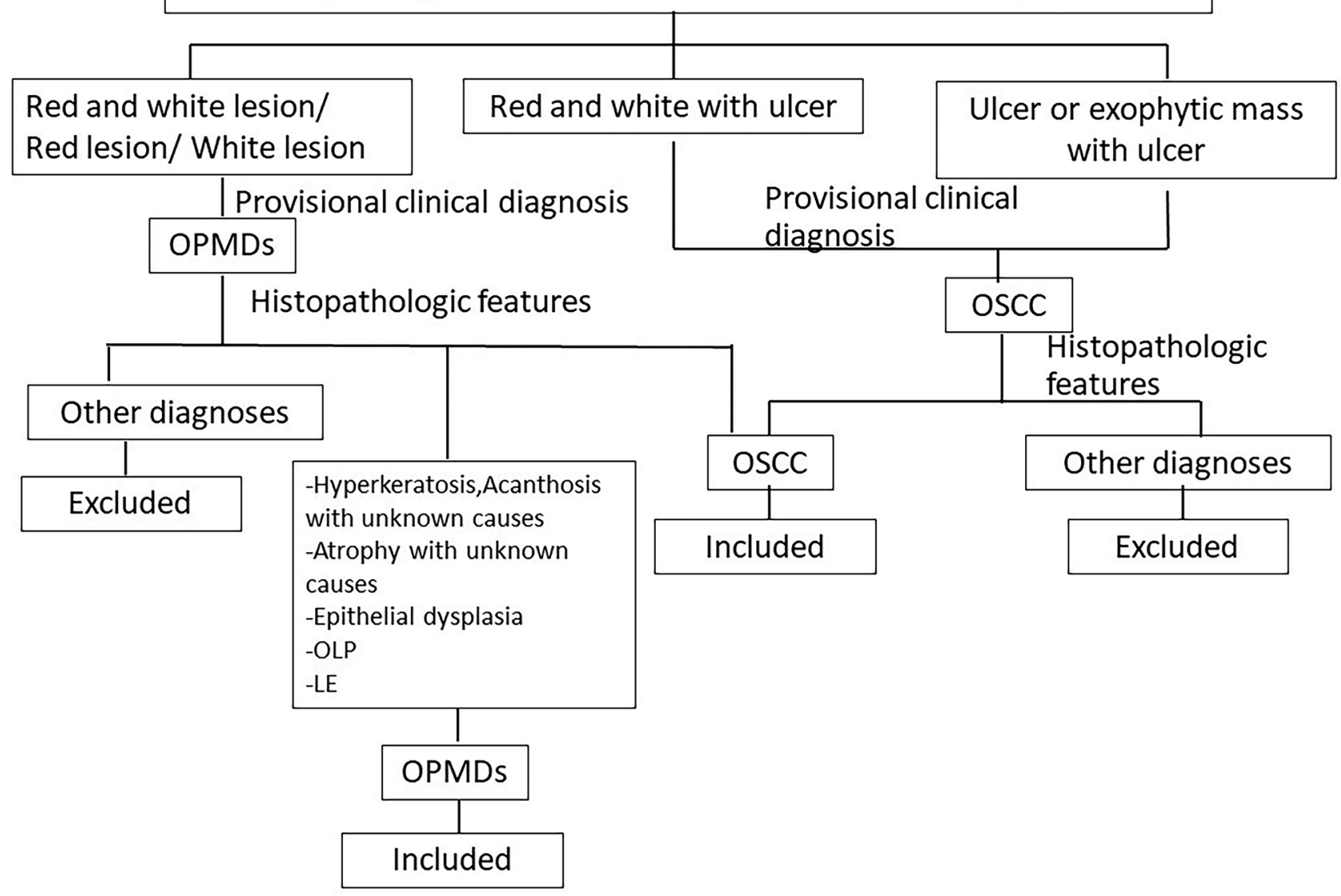

Fig. 1 The screening process of tongue squamous cell carcinoma (SCC) and oral potentially malignant disorder (OPMDs) cases. LE, lupus erythematosus; OLP, oral lichen planus; OSCC, oral squamous cell carcinoma. 
Modification, tongue subsite was classified into six groups: base of tongue dorsal surface, tip and lateral border, ventral surface, other sites of tongue (contiguous or overlapping site of tongue whose point of origin cannot be determined), and tongue, unspecified. ${ }^{7}$

All hematoxylin and eosin-stained slides were reviewed (AI and RJ) for diagnosis confirmation and for histologic classification of tongue SCC and OPMDs. According to the WHO criteria, ${ }^{8}$ histologic grading of tongue SCC was recorded as well, moderately, and poorly differentiated. For histologic evaluation of tongue OPMDs, the presence or absence of epithelial dysplasia and the degree of epithelial dysplasia were determined. In addition, direct immunofluorescence examination was also considered for diagnostic confirmation of OLP and LE.

All data were descriptively analyzed and statistical analysis was performed by SPSS software (version18.0) (SPSS Inc., Chicago, Illinois, United States). Differences between patient's clinical and histopathological aspects were compared using nonparametric chi-squared test. Statistical significance was considered when the $p$-value was $<0.05$.

\section{Results}

\section{Number and Age at Diagnosis of Tongue SCC and OPMDs}

During the 20-year period, 78 cases of tongue SCC $(37.3 \%$ of all OSCC) and 130 cases of tongue OPMDs ( $11.3 \%$ of all OPMDs) were diagnosed. The sex distribution of tongue SCC and OPMDs is presented in - Table 1. The tongue SCC showed a slight male predominance $(\mathrm{M}: \mathrm{F}=1.4: 1)$, whereas a slight female predominance ( $\mathrm{M}: \mathrm{F}=0.7: 1)$ was observed for tongue OPMDs. No significant difference between sexes was found in the prevalence of tongue SCC and OPMDs. According to the age at diagnosis, the highest prevalence of tongue SCC and OPMDs was found in the sixth and seventh decade of life, respectively ( - Fig. 2 ). The mean age of tongue SCC and OPMDs were 43.6 and 52.8 years old, respectively. Of the total number of tongue SCC cases, 14 cases occurred in patients younger than 40 years old, which represented $17.9 \%$ of total tongue SCC. For tongue OPMDs, the highest prevalence of leukoplakia and erythroplakia was observed in the seventh decade of life, while the

Table 1 Tongue SCC and OPMDs reported between 1996 and 2015

\begin{tabular}{|l|l|l|l|l|}
\hline Lesions & Male & Female & Total & $\boldsymbol{p}$-Value \\
\hline OSCC & $46(59 \%)$ & $32(41 \%)$ & 78 & 0.386 \\
\hline OPMDs & $53(40.8 \%)$ & $77(59.2 \%)$ & 130 & 0.388 \\
\hline Leukoplakia & $31(48.4 \%)$ & $33(51.6 \%)$ & 64 & \\
\hline Erythroplakia & $9(34.6 \%)$ & $17(65.4 \%)$ & 26 & \\
\hline OLP & $13(33.3 \%)$ & $26(66.7 \%)$ & 39 & \\
\hline DLE & 0 & $1(100 \%)$ & 1 & \\
\hline
\end{tabular}

Abbreviations: DLE, discoid lupus erythematosus; OLP, oral lichen planus; OPMD, oral potentially malignant disorder; SCC, squamous cell carcinoma.



Fig. 2 Age group distribution (decade of life) of tongue squamous cell carcinoma (SCC) and oral potentially malignant disorder (OPMDs). LE, lupus erythematosus; OLP, oral lichen planus. 
highest prevalence of OLP was seen in the group younger than 40 years old.

\section{Subsites of Tongue SCC and OPMDs}

The number of tongue SCC and OPMDs for each tongue subsite is presented in - Table $\mathbf{2}$. Tip and lateral border of tongue was the most frequently found subsite, accounting for greater than a half of tongue SCC (61.5\%) and OPMDs (56.1\%). Particularly, nearly $80 \%$ of erythroplakia cases were mostly found at the tip and lateral border of tongue. The second most common subsite for tongue SCC and erythroplakia was the ventral surface of tongue while for leukoplakia it was the dorsum of tongue.
Clinical Features of Tongue SCC and OPMDs

- Table 3 shows the distribution of clinical features of tongue SCC and OPMDs. A half of tongue SCC (55.1\%) clinically presented as ulcer or exophytic mass with ulcer. This clinical presentation was significantly greater than that of the other clinical features of tongue SCC $(p<0.025)$. For tongue leukoplakia, the clinical appearance of homogeneous white plaque (69.3\%) was more frequently seen than the nonhomogeneous white plaque $(29.7 \%)$ with significant difference $(p<0.025)$. Tongue erythroplakia cases were frequently presented as rough granular surface (65.4\%) compared with smooth surface $(34.6 \%)$ with significant difference $(p<0.025)$. For the

Table 2 Subsite of tongue SCC and OPMDs

\begin{tabular}{|l|l|l|l|l|l|l|}
\hline \multirow{2}{*}{ Tongue subsites } & OSCC & \multicolumn{5}{|c|}{ OPMDs } \\
\cline { 3 - 7 } & & Leukoplakia & Erythroplakia & OLP & LE & Total \\
\hline Dorsum & $5(6.4 \%)$ & $14(21.9 \%)$ & $1(3.8 \%)$ & $15(38.5 \%)$ & 0 & $30(23.1 \%)$ \\
\hline Ventral surface & $11(14.1 \%)$ & $6(9.4 \%)$ & $4(15.4 \%)$ & $5(12.8 \%)$ & 0 & $15(11.5 \%)$ \\
\hline $\begin{array}{l}\text { Tip and lateral } \\
\text { border }\end{array}$ & $48(61.5 \%)$ & $37(56.7 \%)$ & $20(76.9 \%)$ & $15(38.5 \%)$ & $1(100 \%)$ & $73(56.1 \%)$ \\
\hline Base of tongue & $9(11.5 \%)$ & $4(6.3 \%)$ & 0 & 0 & 0 & $4(3.1 \%)$ \\
\hline $\begin{array}{l}\text { Other sites of } \\
\text { tongue }\end{array}$ & $4(5.1 \%)$ & $3(4.7 \%)$ & $1(3.8 \%)$ & $4(10.3 \%)$ & 0 & $8(6.2 \%)$ \\
\hline $\begin{array}{l}\text { Tongue, } \\
\text { unspecified }\end{array}$ & $1(1.3 \%)$ & 0 & 0 & 0 & 0 & 0 \\
\hline
\end{tabular}

Abbreviations: LE, lupus erythematosus; OLP, oral lichen planus; OPMD, oral potentially malignant disorder; OSCC, oral squamous cell carcinoma.

Table 3 Clinical features of tongue SCC and OPMDs

\begin{tabular}{|c|c|c|c|c|}
\hline \multirow[t]{2}{*}{ Clinical features } & \multicolumn{3}{|c|}{ Number of cases (\%) } & \multirow[t]{2}{*}{$p$-Value } \\
\hline & Male & Female & Total & \\
\hline \multicolumn{5}{|l|}{ OSCC } \\
\hline Red and white lesion & $14(17.9 \%)$ & $9(11.6 \%)$ & $23(29.5 \%)$ & \multirow[t]{3}{*}{$<0.025$} \\
\hline $\begin{array}{l}\text { Red and white lesion with } \\
\text { ulcer }\end{array}$ & $6(7.7 \%)$ & $6(7.7 \%)$ & $12(15.4 \%)$ & \\
\hline $\begin{array}{l}\text { Ulcer or exophytic mass } \\
\text { with ulcer }\end{array}$ & $26(33.3 \%)$ & $17(21.8 \%)$ & $43(55.1 \%)$ & \\
\hline \multicolumn{5}{|l|}{ Leukoplakia } \\
\hline Homogeneous & $26(39.6 \%)$ & 19 (29.7\%) & $45(69.3 \%)$ & \multirow[t]{2}{*}{$<0.025$} \\
\hline Nonhomogeneous & $6(9.4 \%)$ & $13(20.3 \%)$ & $19(29.7 \%)$ & \\
\hline \multicolumn{5}{|l|}{ Erythroplakia } \\
\hline Smooth surface & $1(3.8 \%)$ & $8(30.8 \%)$ & $9(34.6 \%)$ & \multirow[t]{2}{*}{$<0.025$} \\
\hline Rough surface & $8(30.8 \%)$ & $9(34.6 \%)$ & $17(65.4 \%)$ & \\
\hline \multicolumn{5}{|l|}{ OLP } \\
\hline \multicolumn{5}{|l|}{ White lesion } \\
\hline White line & 0 & $1(2.6 \%)$ & $1(2.6 \%)$ & \multirow[t]{4}{*}{$<0.025$} \\
\hline White plaque & $8(20.5 \%)$ & $3(7.6 \%)$ & $11(28.1 \%)$ & \\
\hline Red lesion & 0 & $1(2.6 \%)$ & $1(2.6 \%)$ & \\
\hline Red and white lesion & $5(12.8 \%)$ & $21(53.9 \%)$ & $26(66.7 \%)$ & \\
\hline
\end{tabular}

Abbreviations: OLP, oral lichen planus; OPMD, oral potentially malignant disorder; OSCC, oral squamous cell carcinoma. 
tongue OLP, the majority of cases $(66.7 \%)$ showed the clinical appearance of red and white lesion $(p<0.025)$.

\section{Histopathologic Features of Tongue SCC and OPMDs}

The majority of tongue SCC (82.1\%) was histologically presented as well differentiation, followed by moderate differentiation (17.9\%), whereas poor differentiation was not found (-Table 4). Regarding the presence of epithelial dysplasia, most of tongue OPMDs (64.6\%), except LE, showed epithelial dysplasia, especially all erythroplakia (100\%) and the majority of leukoplakia (81.2\%) (-Table 5). While a half of tongue leukoplakia showed only mild epithelial dysplasia, most of the tongue erythroplakia showed more severity as either moderate (42.3\%) or severe epithelial dysplasia (30.8\%). Only a minority of tongue OLP (15.4\%) showed a presence of epithelial dysplasia.

\section{Clinicopathologic Correlation of Tongue SCC and OPMDs}

For tongue SCC, the most common histologic grading of all clinical features was well differentiation. Any clinical features of tongue SCC were not significantly correlated to histological grade $(p=0.368)$. Although both homogeneous $(80 \%)$ and nonhomogeneous leukoplakia (84.2\%) showed epithelial dysplasia, most of them showed only a mild degree of epithelial dysplasia. Different clinical appearances of tongue leukoplakia were not significantly associated with a presence of epithelial dysplasia $(p=0.699)$. In the erythroplakia cases, moderate epithelial dysplasia was the most common histopathologic diagnosis of both clinical appearances. Chisquared test found no significant correlation between histopathologic finding and clinical presentations of tongue erythroplakia $(p=0.793)$.

\section{Discussion}

In the present study, tongue SCC accounted for $37.3 \%$ of all OSCC. The percentage of tongue SCC falls within the range of several previous reports, mostly ranging between 30 and $46 \%{ }^{9-11}$ Regarding the sex distribution of tongue SCC, we found a slight male predominance ( $\mathrm{M}: \mathrm{F}=1.4: 1)$, that is consistent with previous studies. ${ }^{12,13}$ However, some previous reports clearly showed a male predominance with male to female ratio of 2.3 to $4.3: 1 .{ }^{14,15}$ The peak incidence of tongue SCC in this study fell in the 6th decade of life. The groups of older than 40 years old demonstrated a significantly higher incidence rate than the young age group (40 and below 40 years old), which is consistent with several reports showing a small proportion of OSCC in young patients. ${ }^{4,9,16}$

In this study, we found a low prevalence of OPMDs at the tongue, accounting for $11.3 \%$ of all OPMDs. This figure is lower than previous studies in which tongue OPMD represented

Table 4 Distribution of histologic grade of tongue SCC cases

\begin{tabular}{|l|l|l|l|}
\hline \multirow{2}{*}{ Histologic grade } & \multicolumn{3}{|c|}{ Number (\%) } \\
\cline { 2 - 4 } & Male & Female & Total \\
\hline Well differentiation & $37(47.4 \%)$ & $27(34.7 \%)$ & $64(82.1 \%)$ \\
\hline Moderate differentiation & $9(11.5 \%)$ & $5(6.4 \%)$ & $14(17.9 \%)$ \\
\hline Poor differentiation & 0 & 0 & 0 \\
\hline
\end{tabular}

Abbreviation: SCC, squamous cell carcinoma.

Table 5 Correlation of clinical features and histopathologic diagnosis of tongue OPMDs

\begin{tabular}{|l|l|l|l|l|l|l|}
\hline \multirow{2}{*}{ OPMDs } & \multicolumn{5}{|c|}{ Distopathologic diagnosis } & \multirow{2}{*}{ No dysplasia } \\
\cline { 2 - 6 } & \multicolumn{5}{|c|}{ Megree of dysplasia } & \\
\cline { 2 - 6 } & Mild & Moderate & Severe & Total & \\
\hline Leukoplakia & $32(50.0 \%)$ & $12(18.8 \%)$ & $8(12.5 \%)$ & $52(81.2 \%)$ & $12(18.8 \%)$ & \\
\hline Homogeneous & $23(51.1 \%)$ & $8(17.8 \%)$ & $5(11.1 \%)$ & $36(80.0 \%)$ & $9(20.0 \%)$ & 0.699 \\
\hline Erythroplakia & $9(47.4 \%)$ & $4(21.1 \%)$ & $3(15.7 \%)$ & $16(84.2 \%)$ & $3(15.8 \%)$ & \\
\hline Smooth surface & $7(26.9 \%)$ & $11(42.3 \%)$ & $8(30.8 \%)$ & $26(100 \%)$ & 0 & NA \\
\hline Rough surface & $2(22.2 \%)$ & $4(44.4 \%)$ & $3(33.4 \%)$ & $9(100 \%)$ & 0 & \\
\hline OLP & $5(29.4 \%)$ & $7(41.2 \%)$ & $5(29.4 \%)$ & $17(100 \%)$ & 0 & \\
\hline White lesion & $4(10.3 \%)$ & $1(2.6 \%)$ & $1(2.6 \%)$ & $6(15.4 \%)$ & $33(84.6 \%)$ & \\
\hline Red and white lesion & $2(16.7 \%)$ & 0 & 0 & $2(16.7 \%)$ & $10(83.3 \%)$ & 0.793 \\
\hline Red lesion & $2(7.7 \%)$ & $1(3.85 \%)$ & $1(3.85 \%)$ & $4(15.4 \%)$ & $22(84.6 \%)$ & \\
\hline LE & 0 & 0 & 0 & 0 & 0 & $1(100 \%)$ \\
\hline
\end{tabular}

Abbreviations: LE, lupus erythematosus; OLP, oral lichen planus; OPMD, oral potentially malignant disorder. 
19.7 to $20.7 \%$ of all OPMDs. ${ }^{17,18}$ In contrast to tongue SCC, tongue OPMDs showed a slight female predominance $(\mathrm{M}: \mathrm{F}=$ $0.7: 1)$, which is in agreement with previous studies. ${ }^{18}$ The present study showed that incidence of tongue OPMDs was in 7th decade that is relatively similar to the peak incidence of tongue SCC. Most OPMDs patient in our study were older than 40 years old (82.3\%) that is in agreement with a previous study. ${ }^{18}$ These findings indicate that the elderly people possess a higher risk of tongue cancer development compared with young people.

The most common tongue subsite of tongue SCC was the tip and lateral of tongue (61.5\%). This is in accordance with previous studies revealing that the prevalence of tongue SCC arising at the tip and lateral surface ranged from 61.0 to $82.7 \%{ }^{13,16}$ Consistent with tongue SCC, more than a half of tongue OPMDs (56.1\%) in our study also occurred at the tip and lateral of tongue. Interestingly, in contrast to other OPMDs, in our study tongue OLP was also frequently found at the dorsal surface (38.5\%). This corresponded to a previous study reporting that OLP or oral lichenoid lesions occurred frequently at the dorsal tongue compared with the lateral tongue. ${ }^{19}$

More than a half of tongue SCC (55.1\%) in our study showed the clinical feature of ulcer or exophytic ulcerated mass, which is consistent with a previous study on OSCC. ${ }^{20}$ This finding points that most patients came to seek treatment at more advanced stage of tongue cancer, reflecting a negligence of early cancer detection among Thai patients. Early detection and management of oral cancer can prevent extensive spread and considerably improve the disease prognosis. ${ }^{21}$ For the tongue OPMDs, most of tongue leukoplakia showed the clinical appearance as homogeneous leukoplakia (69.3\%). Within the oral cavity, the high frequency of homogeneous leukoplakia compared with nonhomogeneous shown by previous studies was ranged from 59.6 to $79.3 \% .{ }^{22-24}$

Regarding the histologic grading of tongue SCC, our study demonstrated a very high percentage of well differentiation $(82.1 \%)$ of tongue SCC that is much higher than that of previous reports showing 54.5 to $55.8 \%$ well-differentiated tongue SCC. ${ }^{16,25}$ Interestingly, no case of poor differentiation is found in our study. These results are in accordance with a previous report ${ }^{16}$ and contradict the result of Rai and Ahmed reporting the low percentage of well-differentiated tongue SCC (13.6\%) and high percentage of poorly differentiated tongue SCC $(26.9 \%){ }^{26}$

In the present study, $64.6 \%$ of tongue OPMDs showed the histopathologic feature of epithelial dysplasia. The high proportion of epithelial dysplasia (73\%) among tongue OPMDs has previously been reported by Mello et al. ${ }^{17}$ In comparison to OPMDs at other intraoral sites, dysplastic change of OPMDs at the tongue was considerably higher as reported by previous studies. ${ }^{22,27}$ With regard to each OPMDs, all tongue erythroplakia in the present study showed the histologic feature as epithelial dysplasia, confirming the systemic review of Mello et al showing the highest risk of malignancy for oral erythroplakia. ${ }^{28}$ The percentage of tongue leukoplakia with epithelial dysplasia (81.2\%) in the present study is relatively higher than that of a previous study from India $(68.4 \%)^{22}$ and is remarkably higher than overall leukoplakia at other intraoral sites, ranging from 37 to $62.4 \% .^{22,23,27}$ The susceptibility of the tongue for increased malignancy development may be explained as it is the site of carcinogen pooling in saliva in these food channels and reservoirs. ${ }^{29}$ In addition, tongue epithelium has been proposed to possess an increased turnover rate and are vulnerable to mechanical trauma, microbial biofilm accumulation, as well as superimposed tobacco smoking habits. ${ }^{30}$

Among tongue OPMDs, only $15.4 \%$ of tongue OLP showed epithelial dysplasia; however, this percentage is slightly higher than overall OLP with dysplastic change (10.7\%). ${ }^{31}$ Although the mobile tongue is not the most common site for OLP, OLP involving the tongue carried a higher risk of malignant transformation (51\%) when compared with OLP at other sites. ${ }^{19}$ Regarding the tongue leukoplakia, in the present study different clinical appearances of leukoplakia showed no significant relation to histopathologic feature, which is consistent with a previous study. ${ }^{22}$ However, most previous studies revealed that nonhomogeneous leukoplakia has a higher risk of malignant transformation. ${ }^{23,27}$

\section{Conclusion}

The current study provided a helpful information for clinicians regarding the SCC and OPMDs at the tongue. We found that tongue SCC and OPMDs were particularly found in the elderly population and frequently developed at the tip and lateral of tongue. Tongue SCC was often presented as ulcer or exophytic mass with ulcer, rather than erythroleukoplakia with or without ulcer. Most of tongue OPMDs, in particular erythroplakia and leukoplakia, exhibited epithelial dysplasia. These findings underpin the importance of histopathologic examination of tongue OPMDs and awareness toward early diagnosis of these tongue lesions should be raised for dentists.

\section{Conflict of Interest}

None declared.

\section{References}

$1 \mathrm{Ng} \mathrm{JH}$, Iyer NG, Tan MH, Edgren G. Changing epidemiology of oral squamous cell carcinoma of the tongue: a global study. Head Neck 2017;39(2):297-304

2 Bello IO, Soini Y, Salo T. Prognostic evaluation of oral tongue cancer: means, markers and perspectives (I) Oral Onco 2010;46(9):630-635

3 Warnakulasuriya S. Living with oral cancer: epidemiology with particular reference to prevalence and life-style changes that influence survival. Oral Oncol 2010;46(6):407-410

4 Annertz K, Anderson $\mathrm{H}$, Biörklund $\mathrm{A}$, et al. Incidence and survival of squamous cell carcinoma of the tongue in Scandinavia, with special reference to young adults. Int J Cancer 2002;101(1):95-99

5 Warnakulasuriya S, Johnson NW, van der Waal I. Nomenclature and classification of potentially malignant disorders of the oral mucosa. J Oral Pathol Med 2007;36(10):575-580

6 van der Waal I. Potentially malignant disorders of the oral and oropharyngeal mucosa; terminology, classification and present concepts of management. Oral Oncol 2009;45(4-5):317-323

7 Organization WH, The International Classification of Diseases. 9th edition. Geneva: World Health Organization; 1977 
8 WHO, Histological Typing of Oral and Oropharyngeal Tumours. International Histological Classification of Tumours. Geneva: WHO; 1971

9 Sun $\mathrm{Q}$, Fang $\mathrm{Q}$ Guo S. A comparison of oral squamous cell carcinoma between young and old patients in a single medical center in China. Int J Clin Exp Med 2015;8(8):12418-12423

10 Rikardsen OG, Bjerkli IH, Uhlin-Hansen L, Hadler-Olsen E, Steigen SE. Clinicopathological characteristics of oral squamous cell carcinoma in Northern Norway: a retrospective study. BMC Oral Health 2014;14:103

11 Brandizzi D, Gandolfo M, Velazco ML, Cabrini RL, Lanfranchi HE. Clinical features and evolution of oral cancer: a study of 274 cases in Buenos Aires, Argentina. Med Oral Patol Oral Cir Bucal 2008;13(9):E544-E548

12 Rodrigues PC, Miguel MC, Bagordakis E, et al. Clinicopathological prognostic factors of oral tongue squamous cell carcinoma: a retrospective study of 202 cases. Int J Oral Maxillofac Surg 2014;43(7):795-801

13 Shamloo N, Lotfi A, Motazadian HR, Mortazavi H, Baharvand M. Squamous cell carcinoma as the most common lesion of the tongue in Iranians: a 22-year retrospective study. Asian Pac J Cancer Prev 2016;17(3):1415-1419

14 Vasconcelos MG, Mafra RP, Vasconcelos RG. Medeiros AMCd, Queiroz LMG. Squamous cell carcinoma of the tongue: clinical and morphological analysis of 57 cases and correlation with prognosis. J Bras Patol Med Lab 2014;50:359-363

15 Gueiros LA, Coletta RD, Kowalski LP, Lopes MA. Clinicopathological features and proliferation markers in tongue squamous cell carcinomas. Int J Oral Maxillofac Surg 2011;40(5):510-515

16 Selvamani M, Yamunadevi A, Basandi PS, Madhushankari GS. Prevalence of oral squamous cell carcinoma of tongue in and around Davangere, Karnataka, India: a retrospective study over 13 years. J Pharm Bioallied Sci 2015;7(Suppl 2):S491-S494

17 Mello FW, Melo G, Meurer MI, Rivero ERC. Intraoral potentially malignant disorders in a Brazilian oral pathology service: epidemiological, clinical, and histopathological findings. J Oncol 2018;2018:2325808

18 Hassona Y, Scully C, Almangush A, Baqain Z, Sawair F. Oral potentially malignant disorders among dental patients: a pilot study in Jordan. Asian Pac J Cancer Prev 2014;15(23):10427-10431

19 Fitzpatrick SG, Hirsch SA, Gordon SC. The malignant transformation of oral lichen planus and oral lichenoid lesions: a systematic review. J Am Dent Assoc 2014;145(1):45-56
20 Pires FR, Ramos AB, Oliveira JB, Tavares AS, Luz PS, Santos TC. Oral squamous cell carcinoma: clinicopathological features from 346 cases from a single oral pathology service during an 8-year period. J Appl Oral Sci 2013;21(5):460-467

21 Ramesh R, Sadasivan A. Oral squamous cell carcinoma masquerading as gingival overgrowth. Eur J Dent 2017;11(3):390-394

22 Gopinath D, Thannikunnath BV, Neermunda SF. Prevalence of carcinomatous foci in oral leukoplakia: a clinicopathologic study of 546 Indian samples. J Clin Diagn Res 2016;10(8):ZC78-ZC83

23 Schepman KP, van der Meij EH, Smeele LE, van der Waal I. Malignant transformation of oral leukoplakia: a follow-up study of a hospital-based population of 166 patients with oral leukoplakia from the Netherlands. Oral Oncol 1998;34(4):270-275

24 Scheifele C, Nassar A, Reichart PA. Prevalence of oral cancer and potentially malignant lesions among shammah users in Yemen. Oral Oncol 2007;43(1):42-50

25 Kantola S, Parikka M, Jokinen K, et al. Prognostic factors in tongue cancer - relative importance of demographic, clinical and histopathological factors. Br J. Cancer 2000;83(5):614-619

26 Rai HC, Ahmed J. Clinicopathological correlation study of oral squamous cell carcinoma in a local Indian population. Asian Pac J Cancer Prev 2016;17(3):1251-1254

27 Lee JJ, Hung HC, Cheng SJ, et al. Carcinoma and dysplasia in oral leukoplakias in Taiwan: prevalence and risk factors. Oral Surg Oral Med Oral Pathol Oral Radiol Endod 2006;101(4):472-480

28 Mello FW, Miguel AFP, Dutra KL, et al. Prevalence of oral potentially malignant disorders: a systematic review and metaanalysis. J Oral Pathol Med 2018;47(7):633-640

29 Chen JK, Katz RV, Krutchkoff DJ. Intraoral squamous cell carcinoma. Epidemiologic patterns in Connecticut from 1935 to 1985. Cancer 1990;66(6):1288-1296

30 Narayan TV, Shilpashree S. Meta-analysis on clinicopathologic risk factors of leukoplakias undergoing malignant transformation. J Oral Maxillofac Pathol 2016;20(3):354-361

31 Irani S, Esfahani AM, Ghorbani A. Dysplastic change rate in cases of oral lichen planus: a retrospective study of 112 cases in an Iranian population. J Oral Maxillofac Pathol 2016;20(3):395-399 